\title{
Measurement of water luminescence - a new detection method for neutrino telescopes
}

\author{
The IceCube Collaboration ${ }^{\dagger}$ \\ $\dagger$ http://icecube.wisc.edu/collaboration/authors/icrc17_icecube \\ E-mail: anna.pollmann@uni-wuppertal.de
}

Large-scale neutrino telescopes use water or ice as a target medium. The detection of particles is therefore restricted to those which produce Cherenkov light, bremsstrahlung, or visible secondaries. This excludes the detection of slowly moving, massive particles proposed in theories which go beyond the standard model of particles, including magnetic monopoles.

Simulations show that these particles would be detectable with neutrino telescopes using a little known property of water: highly ionizing particles inducing the production of luminescence after passing through the water and exciting electronic states of the molecules or the lattice.

The few previous measurements of water luminescence show inconsistent results. In addition, there are no measurements with conditions comparable to those present in current large-scale neutrino telescopes. Measurements have therefore been designed that target these particular conditions and enable detailed investigations of the leading uncertainties.

Measurement results are presented and discussed in this contribution where the luminescence of water and ice is induced by $\alpha$-particles. Measurements of warm ice $>-45^{\circ} \mathrm{C}$ are included which has never been considered before.

Corresponding authors: A. Pollmann*1, S. Pieper ${ }^{1}$

${ }^{1}$ Dept. of Physics, University of Wuppertal, 42119 Wuppertal, Germany

35th International Cosmic Ray Conference - ICRC2017-

10-20 July, 2017

Bexco, Busan, Korea

${ }^{*}$ Speaker. 


\section{Luminescence of pure water and ice}

Radio-luminescence is the excitation of transparent media by ionizing radiation resulting subsequently in visible light emission. In 1953 it was shown that pure ice itself emits luminescence light during irradiation [1]. The light was originally thought to originate from impurities in the ice.

Subsequently the effect was investigated in cold ice and liquid water. The measured luminescence yields $G_{\alpha}$, that is the number of photons created per deposited $\alpha$-energy $E_{\alpha}, G_{\alpha}=d N_{\gamma} / d E_{\alpha}$ range from $0.2 \gamma / \mathrm{MeV}$ to $20 \gamma / \mathrm{MeV}$ (summarized in Fig. 5). Measurements of luminescence in warm ice $(>220 \mathrm{~K})$ are not published. The measured luminescence yields vary over orders of magnitude which may arise from different purification methods, saturation effects, or different time resolutions [2,3]. Additionally the luminescence yield of cold ice is dependent on the temperature [4] and the variation of measurement results implies that it is also dependent on the irradiation type or charge due to quenching [5].

The emission spectrum of cold ice luminescence peaks at visible wavelengths with a dominant peak at about $390 \mathrm{~nm}[2,3]$. In addition to the spectrum, the half-lives of the excited states were used to identify the luminescence mechanisms. The measured half-lives range from af ew nanoseconds to several hundred nanoseconds in cold ice [3, 4]. It was deduced that the luminescence originates from certain transitions of excited $\mathrm{OH}^{-}, \mathrm{H}_{3} \mathrm{O}^{+}, \mathrm{H}_{2} \mathrm{O}$, and solved $\mathrm{O}_{2}$ molecules for different temperatures and emission wavelengths. The luminescence mechanisms in liquid water are yet to be identified.

In summary the characteristics of the luminescence of water and ice are not precisely known, especially not in the targeted temperature range from $-40^{\circ} \mathrm{C}$ to $+10^{\circ} \mathrm{C}$.

\section{Luminescence in neutrino telescopes}

The largest neutrino detectors use water and ice as detection media in which charged particles with speeds exceeding $\sim 0.75 c$ induce Cherenkov light emission. Luminescence enables observation of particles at lower speeds, thus it becomes a complementary method of particle detection.

At high energy detectors, such as IceCube, KM3NeT, and Baikal, luminescence enables the detection of slowly moving magnetically or highly electrically charged particles with unprecedented sensitivity [6]. These are particles that are proposed beyond the Standard Model of particle physics, one example are magnetic monopoles which have an equivalent charge of at least $68.5 e$ [7].

Detectors for sub-GeV neutrinos, such as Super- and Hyper-Kamiokande, can detect slowly moving interaction products taking luminescence into account. An example is the kaons that could be produced via the proposed proton decay which, in this case, have an energy below the Cherenkov threshold in water [8].

Luminescence in neutrino detectors would, in addition, refine particle identification methods and the energy calibration of $\mathrm{PeV}$-scale particles. The contribution of photons from luminescence can be up to $10 \%$ of the amount of Cherenkov photons when assuming an energy deposition in the $\mathrm{PeV}$ range.

At sub-GeV energies, particle identification is based on discrimination of sharp edged rings induced by muons on the one hand and blurred rings by electron induced cascades on the other 
hand. Isotropic contributions, such as Rayleigh scattering and luminescence, hamper the discrimination power of reconstruction tools. The delayed emission of luminescence photons compared to Cherenkov photons adds to this reconstruction uncertainty. The particle identification could be improved if the features of luminescence could be included into simulation and reconstruction algorithms.

The contribution of salt water luminescence, as in the sea for KM3NeT or the salt phase of the Sudbury Neutrino Observatory and Super-Kamiokande-Gd, could be even larger [9, 10]. At detectors with artificial media, salt is added to enhance the neutron capture cross section and the delayed light signal. Identifying the coincidence of a positron with a delayed neutron capture significantly improves the sensitivity for Supernova events. However, the delayed neutron capture signal has to be discriminated from long decay times of luminescence.

Apart from one measurement by Baikal [11], there are no measurements covering the conditions of the large neutrino telescopes KM3NeT, ANTARES, IceCube, Super-Kamiokande(-Gd), Hyper-Kamiokande, or SNO and previous laboratory measurements vary significantly. Thus, luminescence of water and ice needs to be measured and characterized precisely for a future application at water and ice based particle detectors.

\section{Measurement setup}

A setup was developed to measure luminescence induced by $\alpha$-particles emitted by an ${ }^{241} \mathrm{Am}$ source in ultra-purified water and ice, see Fig. 1. The light emission is recorded by a PMT. Measurements are performed inside a dark box which is placed in a freezer, that is used for temperature control.
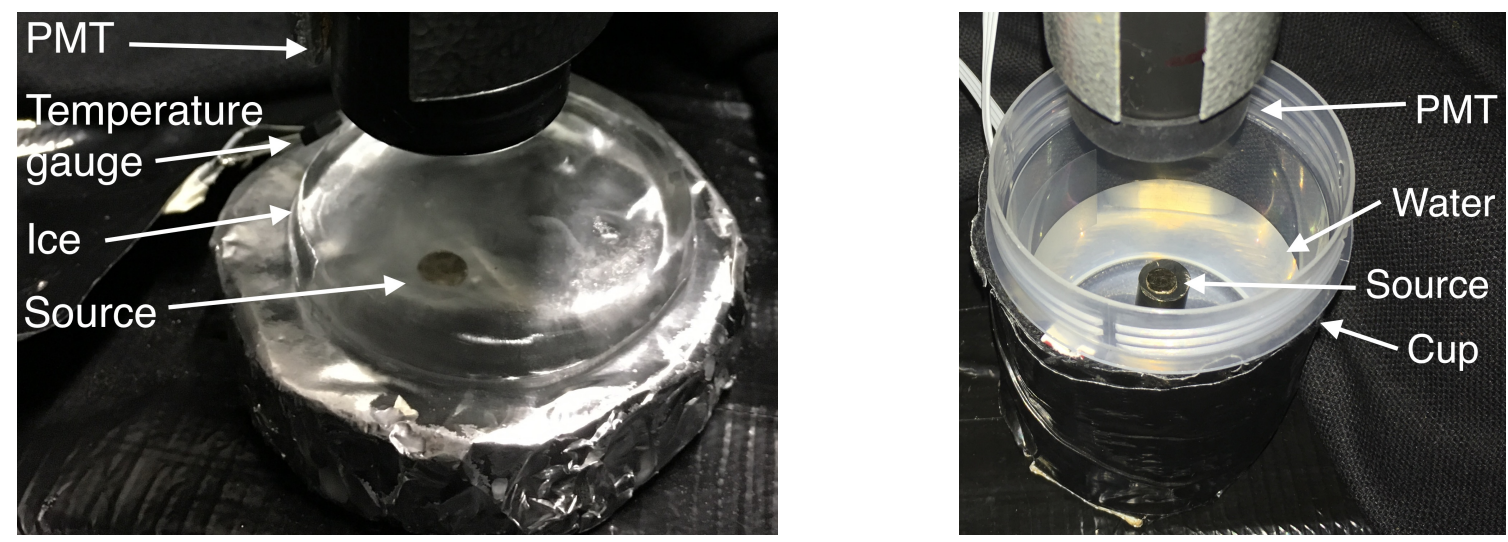

Figure 1: Photograph of the setup for measuring the luminescence yield of ice (left) and water (right). The distance from PMT to sample surface was increased to take the picture.

For measurements above $0{ }^{\circ} \mathrm{C}$, the ${ }^{241} \mathrm{Am}$ source is placed on a stainless steel cylinder inside the water sample that is stored in a sterile polypropylene cup. The single-use cup is manufactured for liquid biological samples of about $100 \mathrm{ml}$ volume.

Bubble and crack free ice was grown by freezing purified and vacuum degassed water at $\approx$ $-15^{\circ} \mathrm{C}$ within a sterile cup which was placed into a water-filled styrofoam box. The process was stopped after $\approx 12$ hours when only $\approx 1 \mathrm{~cm}$ of ice was grown at the water surface. The lower ice 
surface was straightened by placing it for $\approx 1$ minute on a metallic surface at room temperature. The source was attached to the sterile upper ice surface which was then frozen at $\approx-20^{\circ} \mathrm{C}$ for 10 minutes. The ice was then placed upside-down on a cleaned aluminum foil to reflect photons upwards as shown in Fig. 2.

The purified water used in these measurements has a resistance of $>18 \mathrm{M} \Omega \mathrm{cm}$ and total organic carbon (TOC) of $<10 \mathrm{ppb}$. All materials which have contact with the water, apart from the sterile cups, are cleaned by placing in purified water for at least several hours after conventional cleaning.

The source characteristics were measured with the same setup using a known scintillator and a ${ }^{241} \mathrm{Am}$ calibration source. The ${ }^{241} \mathrm{Am}$ source used has a surface emission rate of $(51 \pm 5) \mathrm{kBq}$ for $\alpha$-particles over $2 \pi \mathrm{sr}$. The emission energy of the $5.486 \mathrm{MeV} \alpha$-particles was measured to be (4.4 \pm 0.4$) \mathrm{MeV}$ because the ${ }^{241} \mathrm{Am}$ is covered with a thin layer of gold by the manufacturer. $\alpha$-particles of $4.4 \mathrm{MeV}$ loose all energy within $\approx 11 \mu \mathrm{m}$ in ice. X-rays are also produced from the source with highest intensities at $13.9 \mathrm{keV}(37 \%)$ and $59.54 \mathrm{keV}(35.9 \%)$ [12].
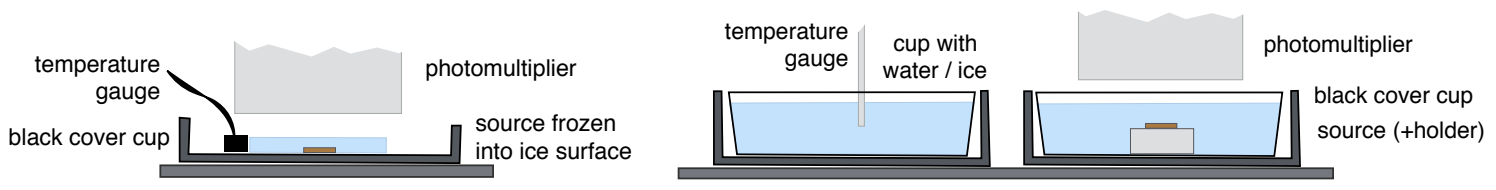

Figure 2: Schematic drawing of the setup for measuring the luminescence yield of ice (left) and water (right).

Photons, which might be created in the setup, are then detected by a photomultiplier tube (PMT) that was selected for single photon counting [13] and placed just above the water and ice surfaces over the source as shown in Fig. 1. In case of water this was done to avoid contaminations inside the purified water samples. The PMT was measured to be sensitive from $250 \mathrm{~nm}$ to $650 \mathrm{~nm}$. It is operated with positive high voltage to avoid charge collection at the cathode. The efficiency of the PMT per wavelength was measured in comparison with a calibrated diode. It is maximal with $(27.2 \pm 1.3) \%$ at wavelengths of $(380-390) \mathrm{nm}$. It varies $\pm 1 \%$ within an inner radius of $6 \mathrm{~mm}$ of the photo-cathode and drops afterwards approximatly linearly.

The non-amplified signal of the PMT is read out with a digital oscilloscope [14]. The peak value per triggered $100 \mathrm{~ns}$ sample is saved as the amplitude. The rate is calculated by dividing the number of triggered hits by the required recording time whereas the latter is obtained from the PC which drives the oscilloscope. The recording time was ensured to exceed $1 \mathrm{~s}$ to ensure small uncertainty on the measurement duration. The dead-time of the oscilloscope is lower than $1 \mu \mathrm{s}$ and the reproduction of signal generator rates was checked up to $100 \mathrm{kHz}$. Other parameters are monitored to ensure stable conditions, i.e. room temperature, freezer temperature, environmental electromagnetic noise, PMT high voltage, CPU workload and room light.

\section{Background}

The contributions of different background sources, shown in Fig. 3, were measured to isolate the signal induced by $\alpha$-particles in water, i.e. the PMT dark rate, environmental light, natural radioactivity, cosmic rays, $\mathrm{X}$-rays from the source and electronic noise. 


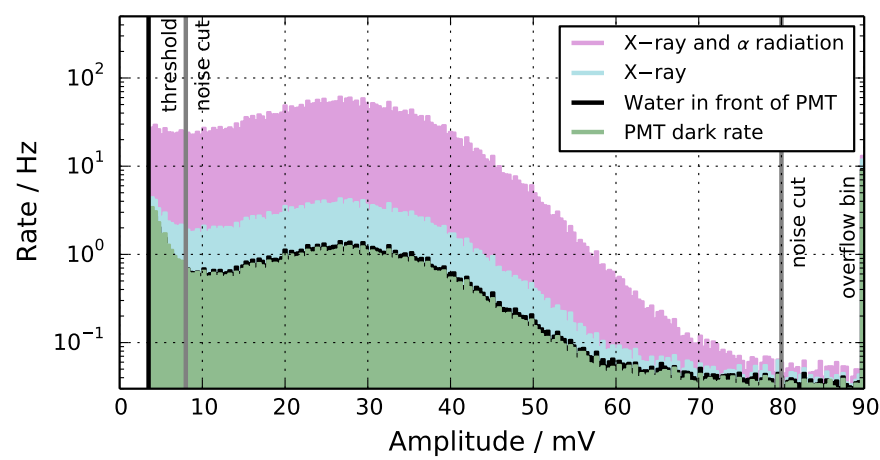

Figure 3: Background and signal contributions of luminescence induced with $\alpha$-particles from ${ }^{241} \mathrm{Am}$ measured at $25^{\circ} \mathrm{C}$ in ultra-purified water. The trigger threshold is at $4 \mathrm{mV}$. The last bin is an overflow bin. The main peak matches the position of the single photon peak. Noise was reduced by discarding events with peak amplitude below $8 \mathrm{mV}$ and above $80 \mathrm{mV}$. The experimental procedure as well as the interpretation are explained in the text.

The measured PMT dark rate, shown in Fig. 3, includes negligible environmental light. A cup of water was placed in front of the PMT to measure the contribution of natural radioactivity in water, cup, and surroundings as well as cosmic ray interactions in the water.

The X-rays emitted by ${ }^{241} \mathrm{Am}$ undergo Compton scattering in media producing free low energetic electrons which produce luminescence. The light yield of Compton scattering in the PMT glass dominates over the light yield from scattering in water or other parts of the setup. The latter was determined by covering the PMT cathode with a black lid and placing the source beneath a black sheet of plastics in front of the PMT. The measured signal is well above the dark rate and shows a temperature dependence, see Sec. 5. The regarding light yield of glass scintillation is roughly $3 \gamma / \mathrm{MeV}$ which is compatible with a previous glass scintillation measurement [15]. The Compton scattering in the cup or the ice holder was measured to be negligible. The summed contribution of X-rays, shown in Fig. 3, was measured by placing the source into the water (or below the ice) under a cover of black plastics. The contribution of $\alpha$-irradiation of water over all background is then measured with the source in water (or frozen onto the lower surface of the ice) without any cover.

As shown in Fig. 3, the contribution of $\alpha$-irradiation to the total light emission is significantly stronger than all background contributions which are subtracted in further calculations. For further analysis, all pulses with a peak amplitude between $8 \mathrm{mV}$ and $80 \mathrm{mV}$, which translates to approximately $(0.25-2.5) \mathrm{PE}$, are kept to reduce electronic and environmental noise.

\section{Temperature dependence}

The temperature of the setup was adjusted using a freezer or by warming/cooling to/from room temperature outside the operational temperatures. The PMT base was covered with a thin plastic bag to prevent condensation on the electronics causing instable rates. The temperature of the water sample was estimated by measuring the temperature in the middle of a dummy sample which was treated identically to the irradiated sample and placed directly next to it. A temperature gauge was 

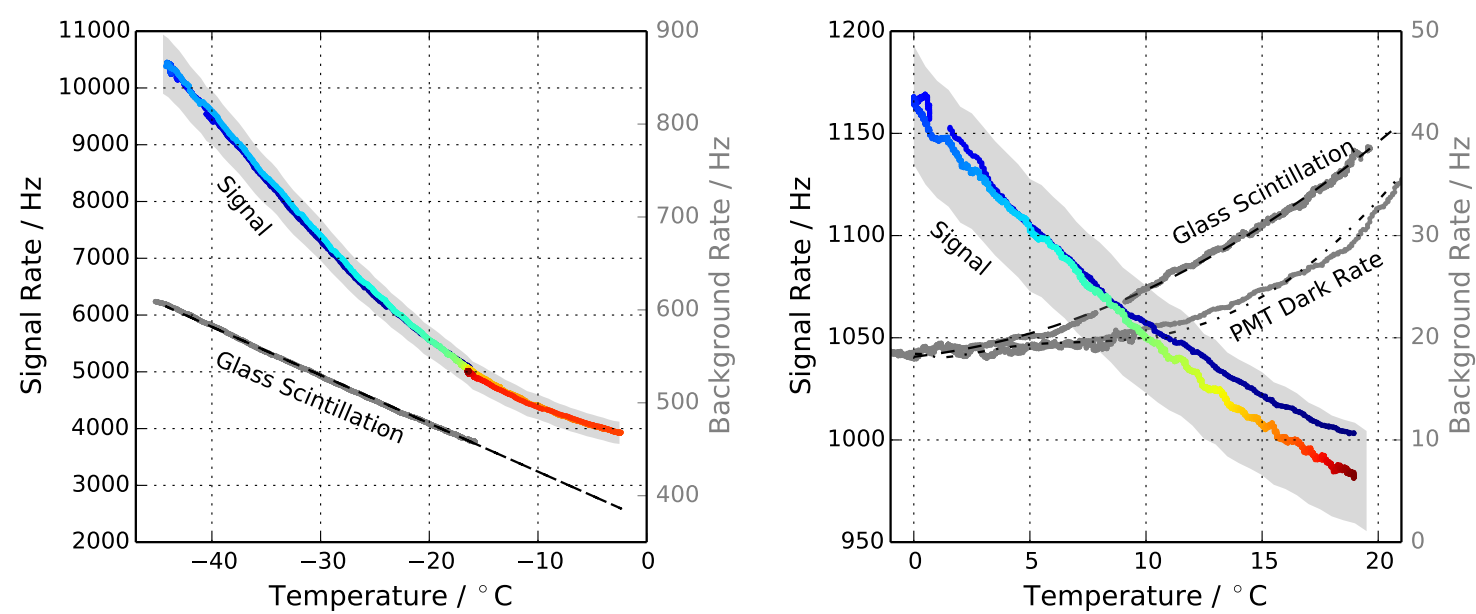

Figure 4: Temperature dependence of the light emission from $\alpha$-irradiated ice (left) and water (right). The color scale shows the measurement time from blue over green and yellow to red. Gray bands indicate the measurement uncertainty of the rate. The temperature dependence of the setup, that is also shown, has been already subtracted from the signal shown here. The background rates are fit with a linear $\left(<0^{\circ} \mathrm{C}\right)$ and polynomial $\left(>0^{\circ} \mathrm{C}\right)$ distribution. Due to different geometries the absolute rates are not comparable.

frozen onto the side surface of the ice sample to measure its temperature. Data was taken during cooling and warming to ensure the absence of a significant hysteresis.

The temperature dependence of the PMT dark rate, the ice without source (to exclude triboluminescence), the water and ice under X-ray irradiation, and the glass scintillation was measured and subtracted from the rates shown in Fig. 4. A non-linear temperature dependence was measured which is slight for water and strong for ice irradiated by $\alpha$-particles.

\section{Discussion}

A clear signal of single photons induced by $\alpha$-irradiation of water and ice was isolated over background. This signal shows a temperature dependence in water and ice which is stronger than or inverted to all measured temperature dependencies of the setup. The Cherenkov threshold of $0.76 c$ in ice is at $\approx 270 \mathrm{keV}$ for electrons. $\alpha$-particles with an energy of $5 \mathrm{MeV}$ have a speed of $0.05 c$ and the respective $\delta$-electrons have a maximal energy of $\approx 3 \mathrm{keV}$ [16]. Thus, no Cherenkov light is expected in this measurement. Additionally, Cherenkov light depends only on the speed of the incident particle and the medium's refractive index [17]. The latter is only slightly temperature dependent which does not explain the temperature dependence of the measured light.

The influence of solubles, originating from the materials in touch with the water samples, is tested by monitoring the signal rate directly after insertion of larger amounts of cleaned material into water for two days. No measurable effect was seen.

Thus, it is concluded that radio-luminescence of water and ice itself, induced by $\alpha$-particles, was measured. At least one of the transitions from the excited electronic states in each, water and ice, shows a temperature dependency. For further conclusions the emission spectra and decay kinetics have to be measured. 


\section{Luminescence yield}

The luminescence yield was calculated from the rate of measured single photons which was cleaned from background and noise as described above. A Monte Carlo simulation was executed to estimate the number of emitted photons which can be seen by the PMT. The measured ice/water thickness, its distance to the PMT, the refraction at the ice/water - air surface and reflection at both, the gold layer of the source and the sample - air surface was calculated for each photon. The measured PMT spectral, and radial efficiency was also included. A uniform emission spectrum between $250 \mathrm{~nm}$ and $650 \mathrm{~nm}$ was assumed. The regarding uncertainty was included by assuming $100 \mathrm{~nm}$ wide peaks in this range. A more precise estimation requires the measurement of the emission spectrum and subsequent optical calibration.

The estimated luminescence yield for the given wavelength range is shown in Fig. 5. It ranges from $0.42_{-0.25}^{+0.19} \gamma / \mathrm{MeV}$ to $0.50_{-0.29}^{+0.23} \gamma / \mathrm{MeV}$ for water and from $0.54_{-0.83}^{+0.64} \gamma / \mathrm{MeV}$ to $1.25_{-0.31}^{+0.24} \gamma / \mathrm{MeV}$ for ice. A discontinuity between the measurements at $0{ }^{\circ} \mathrm{C}$ is expected due to the differences between electronic excitations in lattice and molecules in liquids. This result is in tension with two measurements of water luminescence [18] using distilled water which is usually of significantly lower purity than the water used in this study. The third measurement, which is within uncertainties in agreement with this result, is an in-situ measurement in the Baikal lake [11].

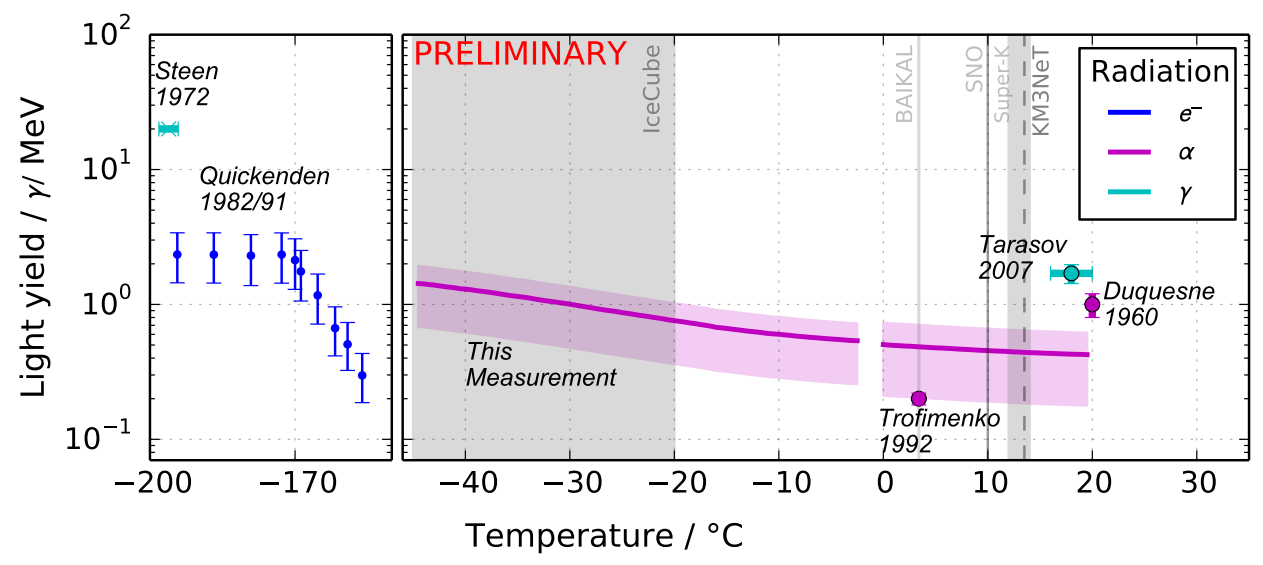

Figure 5: The result of this measurement is shown in comparison to measured luminescence yields of cold ice, warm ice, and liquid water induced by different kind of irradiations, taken from Refs. [2, 18, 19, 11]. Older measurements for cold ice are summarized in Ref. [2]. The water and ice temperatures of neutrino detectors are shown as vertical bands $[9,20]$. In addition to the values above, there is a recent measurement of water luminescence induced by $\alpha$-particles, protons, and carbon-ions which gives relative values only [21].

\section{Summary and outlook}

The luminescence of ice $>-45^{\circ} \mathrm{C}$ was measured for the first time. This opens up the prospect of using luminescence as a detection method for the IceCube neutrino telescope. It was shown in simulation studies that ice luminescence can be used for magnetic monopole detection if the 
luminescence yield exceeds $0.2 \gamma / \mathrm{MeV}$ [6] which is the case. However, the yield varies with ice temperature which is not constant through the depth of the detector volume.

The first measurement of the temperature dependence of water and ice ()$\left.>-45^{\circ} \mathrm{C}\right)$ luminescence was achieved. A temperature dependence was seen, however, this does not explain the tension between previous measurements. Further studies are planned to measure the influence of solubles to derive a value usable for neutrino detectors using liquid water as medium.

Further measurements are planned at ion accelerators to measure the emission spectra, decay kinetics and quenching behavior for a full characterization of water and ice luminescence. Water and ice samples from the detector sites will be used for these measurements in addition to ultrapurified water to take their properties into account.

\section{References}

[1] E. H. Belcher, Proc. R. Soc. Lond. A 216 (1953) 90-102.

[2] T. I. Quickenden, S. M. Trotman, and D. F. Sangster, J. Chem. Phys. 77 (1982) 3790.

[3] S. M. Trotman, T. I. Quickenden, and D. F. Sangster, J. Chem. Phys. 85 (1986) 2555.

[4] T. I. Quickenden et al., J. Chem. Phys. 95 (1991) 8843.

[5] V. Langford et al., Acc. Chem. Res. 33 (2000) 665-671.

[6] A. Pollmann. Proceedings of ICNFP'16. arXiv:1610.06397.

[7] P. Dirac, Proc. Roy. Soc. A 133 (1931) 60-73.

[8] Y. Hayato et al., Phys. Rev. Lett. 83 (1999) 1529.

[9] P. Bagley et al., KM3NeT - Technical Design Report, 2009. http://inspirehep.net/record/1366114/files/TDRKM3NeT.pdf.

[10] C. Xu et al., J. Phys. Conf. Ser. 718 (2016), no. 6 062070. ; B. Aharmim et al., Phys. Rev. C 72 (2005) 055502.

[11] V. Aynutdinov et al., Astropart. Phys. 29 (2008) 366 - 372. Original description in I. I. Trofimenko, preprint INR 765/92 (in Russian).

[12] M. S. Basunia, Nuclear data sheets 107, 3323, 2006.

[13] Photomultiplier Tube R1924P by HAMAMATSU Photonics K.K., 2017.

[14] PicoScope 6404B by Pico Technology, 2017.

[15] K. Helbing et al. AMANDA internal note IR/2030701.

[16] J. Beringer et al., Phys. Rev. D 86 (2012) 323. Ch. 30.

[17] E. Tamm and M. Frank, Dokl. Akad. Nauk SSSR (Akad. of Science of the USSR) 14 (1937) 107.

[18] M. Duquesne and I. Kaplan, Phys. Radium 21 (1960) 708. (in French); M. D. Tarasov et al., Instrum. Exp. Tech. 50 (2007), no. 6 761-763.

[19] H. B. Steen, O. I. Sorensen, and J. A. Holteng, Int. J. Radiat. Phys. Chem. 4 (1972) 75-86.

[20] A. Achterberg et al., Astropart. Phys. 26 (2006) 155-173. ; V. Aynutdinov et al., Nucl. Instr. Meth. A 602 (2009) 14-20. ; J. Boger et al., Nucl. Instr. Meth. A 449 (2000) 172-207. ; S. Fukuda et al., Nucl. Instr. Meth. A 501 (2003) 418-462.

[21] S. Yamamoto, in Proc. SPIE, vol. 10049. doi:10.1117/12.2255648. 